Randomised controlled trial

\section{Naloxegol increases frequency of bowel movements and combats inadequate response to laxatives}

10.1136/ebmed-2014-110071

\section{Peter Holzer}

Institute of Experimental and Clinical Pharmacology, Medical University of Graz, Graz, Austria

Correspondence to: Dr Peter Holzer, Institute of Experimental and Clinical Pharmacology, Medical University of Graz, Universitätsplatz 4, Graz A-8010, Austria; peter.holzer@medunigraz.at

Commentary on: Chey WD, Webster L, Sostek M, et al. Naloxegol for opioid-induced constipation in patients with noncancer pain. N Engl J Med 2014;370:2387-96.

\section{Context}

The therapeutic action of opioid analgesics is compromised by peripheral adverse effects, among which constipation is the most disabling, as laxatives often fail to provide satisfactory relief. ${ }^{1}$ To address this problem, opioid receptor antagonists with limited systemic bioavailability (retarded naloxone) or a peripherally restricted site of action (alvimopan, methylnaltrexone) have been developed. Their use in managing opioid-induced constipation (OIC), however, is limited by narrow indication and subcutaneous administration (methylnaltrexone), approval only for short-term use because of safety concerns (alvimopan) or a fixed combination of retarded naloxone with oxycodone. ${ }^{2}$ Naloxegol is a peripherally acting $\mu$-opioid receptor antagonist under development as an oral, daily administered drug for the treatment of OIC. ${ }^{3}$ Chemically, it is a pegylated derivative of naloxone, the PEG moiety making the compound a substrate for P-glycoprotein transporters which limit drug entry into the central nervous system (CNS). ${ }^{3}$

\section{Methods}

The paper describes two identical randomised, double-blind, placebocontrolled phase 3 trials involving a total of 1352 outpatients suffering from non-cancer pain, experiencing pain relief by stable oral opioid medication and reporting OIC, defined by less than three spontaneous bowel movements (SPMs) per week accompanied by other symptoms. Participants were assigned to receive placebo or a daily dose of 12.5 or $25 \mathrm{mg}$ naloxegol for 12 weeks, and stratified such that at least 50\% of the participants in each treatment arm had an inadequate response to laxatives. Achievement of three or more SPMs per week was the primary end point. Secondary end points included three or more SPMs per week among patients who previously did not respond to laxatives.

\section{Findings}

Naloxegol significantly enhanced response rates for the primary and secondary end points in a dose-dependent manner relative to placebo. In one trial, a significant increase in the response rate was seen with both doses of naloxegol, while in the other trial only the $25 \mathrm{mg}$ dose had a statistically significant effect. The number needed to treat at $25 \mathrm{mg}$ was
6.7 in study 1 and 9.7 in study 2. A similar dose-related effect of naloxegol was noted for secondary end points, especially response rate in patients who had responded inadequately to laxatives before enrolment. The incidence of adverse events, notably diarrhoea and abdominal pain, was highest in the $25 \mathrm{mg}$ dose group.

\section{Commentary}

The results of the two phase 3 trials confirm and extend a phase 2 study of patients with OIC in whom a 4-week treatment with daily doses of 5 , 25 and $50 \mathrm{mg}$ naloxegol increased the number of weekly SPMs in a dosedependent fashion. ${ }^{3}$ In addition, the current data indicate that patients who have an inadequate response to laxatives benefit from the use of naloxegol. The absence of withdrawal symptoms and pain exacerbation indicate that the drug ameliorates OIC by a peripheral site of action but does not interfere with the analgesic effect of opioid analgesics within the CNS. Naloxegol is thus set to join the class of peripherally active opioid receptor antagonists that do not enter the CNS. Naloxegol may claim significant advantages over other members of the class in terms of indication, route of administration, flexibility of use and safety; however, it has not yet been proven to be equally efficacious in attenuating OIC. This issue is difficult to judge since no comparative trial results are yet available, while clinical trials involving individual drugs are difficult to compare because they differ in their clinical end points or in the way they were analysed.

Apart from opioid-receptor-related adverse events (diarrhoea, abdominal pain) which can be interpreted as manifestations of gastrointestinal opioid withdrawal, naloxegol appears to be fairly safe over a 12-week treatment period. ${ }^{2}$ At the dose of $25 \mathrm{mg} /$ day, naloxegol lacks a clinically relevant effect on cardiac repolarisation in healthy volunteers. ${ }^{4}$ Although naloxegol is primarily cleared by a hepatic route, mild or moderate hepatic impairment has minimal effects on the pharmacokinetics and safety of the drug, while renal impairment may necessitate dose adjustments. $^{56}$

\section{Competing interests None.}

Provenance and peer review Commissioned; internally peer reviewed.

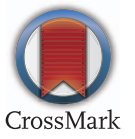

\section{References}

1. Holzer P. Opioid antagonists for prevention and treatment of opioid-induced gastrointestinal effects. Curr Opin Anaesthesiol 2010;23:616-22.

2. Holzer P. Non-analgesic effects of opioids: management of opioid-induced constipation by peripheral opioid receptor antagonists: prevention or withdrawal? Curr Pharm Des 2012;18:6010-20.

3. Webster L, Dhar S, Eldon M, et al. A phase 2, double-blind, randomized, placebo-controlled, dose-escalation study to evaluate the efficacy, safety, and tolerability of naloxegol in patients with opioid-induced constipation. Pain 2013;154:1542-50.

4. Gottfridsson C, Carlson G, Lappalainen J, et al. Evaluation of the effect of naloxegol on cardiac repolarization: a randomized, placebo- and positive-controlled crossover thorough QT/QTc study in healthy volunteers. Clin Ther 2013;35:1876-83.

5. Bui K, She F, Sostek M. The effects of mild or moderate hepatic impairment on the pharmacokinetics, safety, and tolerability of naloxegol. J Clin Pharmacol Published Online First: 19 Jun 2014. doi:10.1002/jcph.348

6. Bui K, She F, Sostek M. The effects of renal impairment on the pharmacokinetics, safety, and tolerability of naloxegol. J Clin Pharmacol Published Online First: 19 Jun 2014. doi:10.1002/jcph.349 